\title{
SELEÇÃO DE CLONES DE CAJUEIRO COMUM PELO MÉTODO EM “TANDEM"E ÍNDICE DE CLASSIFICAÇÃO
}

\author{
Selection of common cashew clones by tandem method and rank index
}

\author{
João Rodrigues de Paiva ${ }^{1}$, José Jaime Vasconcelos Cavalcanti' ${ }^{1}$ Levi de Moura Barros ${ }^{1}$, \\ Márcio Cleber de Medeiros Corrêa ${ }^{2}$, Maria Clideana Cabral Maia², Alano Bastos Costa Filho ${ }^{3}$
}

\begin{abstract}
RESUMO
Com o objetivo de selecionar clones de cajueiro comum e avaliar a eficiência da metodologia do índice da soma de classificação no melhoramento da cultura foi instalado, em 1999, um experimento em área de produtor, no município de Beberibe, CE, no delineamento de blocos ao acaso com 40 clones, três repetições e cinco plantas por parcela, no espaçamento de $10 \mathrm{~m} \times 10 \mathrm{~m}$. Os clones foram avaliados, durante cinco anos, para altura de planta $(\mathrm{m})$ e diâmetro da copa $(\mathrm{m})$. A avaliação da produção de castanha $(\mathrm{kg} / \mathrm{planta/}$ safra) foi feita em três safras. A maior produtividade de castanha foi para o clone Comum 30, com $885 \mathrm{~kg} \cdot \mathrm{ha}^{-1} \mathrm{e} 1.117 \mathrm{~kg} . \mathrm{ha}^{-1}$, respectivamente na primeira e segunda safra, enquanto que o clone Comum 21 se destacou no terceiro ano de produção com 1.299 kg.ha ${ }^{-1}$. Pela avaliação conjunta do porte das plantas, produção de castanha, performance fenotípica do clone e características tecnológicas da amêndoa, os clones Comum 18, Comum 21, Comum 28, Comum 30, Comum 31 e Comum 36 foram selecionados para teste em larga escala. A seleção de clones de cajueiro comum através do índice da soma de classificação revelou-se inapropriada quando comparada ao método em "tandem".
\end{abstract}

Termos para indexação: Anacardium occidentale, método de seleção, produção.

\begin{abstract}
The objective of this work was to select clones of common cashew and to evaluate the efficiency of the rank index in the cashew improvement. The trial was set up in May 1999 using a randomized complete block design with 40 clones, 3 replications and 5 plants per plot, spaced in $10 \mathrm{~m} \times 10 \mathrm{~m}$. Plant height $(\mathrm{m})$ and canopy diameter $(\mathrm{m})$ were measured for five years. Nut yield control $(\mathrm{kg} /$ plant/year) was done for three years. The Common clone 30 showed the largest nut yield, $885 \mathrm{~kg} \cdot \mathrm{ha}^{-1}$ and $1.117 \mathrm{~kg} \cdot \mathrm{ha}^{-1}$ for the first and second year of evaluation, respectively. Considering the caracteristics plant vigour, nut yield and kernel quality used together as reference of population average, the clones Common 18, Common 21, Common 28, Common 30, Common 31 and Common 36 were selected as the most promising for test in wide scale and local commercial plantation. The selection of common cashew clones by rank index was inefficient in relation to the "tandem" method.
\end{abstract}

Index terms: Anacardium occidentale, selection method, yield.

(Recebido em 9 de março de 2006 e aprovado em 12 de dezembro de 2006)

\section{INTRODUÇÃO}

O agronegócio caju gera emprego e renda para milhares de pessoas e divisas cambiais para os países produtores e exportadores, quase todos dependentes do negócio agrícola nas suas economias. De acordo com a FAO (2005), o Vietnã é o maior produtor de castanha com cerca de 640.000 t/ano em 2004, seguido da Índia (460.000), Brasil (223.941 t) e Nigéria (186.000 t). A evolução da produção de castanha de caju nos últimos 30 anos mostra que, a partir do início dos anos noventa, alguns países asiáticos assumiram lugar de importância entre os produtores.

No Brasil, apesar de sua importância sócioeconômica, esta exploração sempre esteve à margem do emprego de tecnologias, ocasionando redução da produtividade. As baixas produtividades vêm repercutindo em toda a cadeia, do segmento produtivo à industrialização, na falta de matéria-prima de qualidade para a indústria. Portanto, há necessidade de aumentar a lucratividade do setor produtivo uma vez que as produtividades auferidas atualmente não remuneram o produtor, pondo em risco a viabilidade do agronegócio estabelecido (LEITE \& PAULA-PESSOA, 2002).

Os atuais clones de cajueiro recomendados para o plantio comercial são todos do tipo anão precoce. Entretanto, a inexistência de clones do tipo comum, de certa forma, reflete a dificuldade de obtenção e seleção de plantas que reúnam vários atributos de valor econômico em um só indivíduo. A utilização de metodologias que permitem selecionar plantas, considerando vários caracteres simultaneamente, deve ser analisada visando o seu emprego futuro como "ferramenta" auxiliar no melhoramento do cajueiro.

1Engenheiro Agrônomo, D.Sc., Embrapa Agroindústria Tropical - Cx. P. 3761 - 60511-110 - Fortaleza, CE - paiva@cnpat.embrapa.br ²Engenheiro(a) Agrônomo, D.Sc., UFC/CCA - Cx. P. 60451-970 - 60455-900 - Fortaleza, CE - mcleber@ufc.br ${ }^{3}$ Engenheiro Agrônomo, D. Sc., Bolsista DTI/CNPq 
Paiva et al. (2002) mostraram a eficiência da metodologia de Mulamba \& Mock (1978), descrita por Cruz \& Regazzi (1997), na seleção de progênies de acerola, em comparação ao método tradicional de seleção entre e dentro, destacando a facilidade e praticidade de execução. Silva et al. (2003) também utilizaram essa metodologia na seleção de clones superiores no programa de retrocruzamento de batata com resultados favoráveis.

Realizou-se este trabalho com objetivo de selecionar clones de cajueiro comum, com atributos de porte e produção de castanhas adequados ao cultivo comercial, e avaliar o uso da metodologia do índice da soma de classificação no melhoramento da cultura.

\section{MATERIAL E MÉTODOS}

O experimento foi instalado em março de 1999 na Fazenda Jacaju, pertencente à Cia. de Óleos do Nordeste (CIONE), localizada no km 17 da BR 304 no município de Beberibe, CE.

Os clones de cajueiro comum foram obtidos pela enxertia de plantas com características de porte médio da planta, elevada produção de castanha e peso da amêndoa. Foi utilizado o delineamento de blocos ao acaso com 40 tratamentos, três repetições, cinco plantas por parcela, no espaçamento de $10 \mathrm{~m}$ x $10 \mathrm{~m}$ e bordadura de contorno envolvendo todo o experimento. A área total do experimento foi de 7,04 ha.

Os tratos culturais aplicados no experimento foram os mesmos utilizados na fazenda para o cultivo do cajueiro comum em condições de sequeiro (OLIVEIRA, 2004). Os clones foram avaliados, durante cinco anos, para altura de planta (m) e diâmetro de copa (m). O controle da produção de castanha (kg/planta/safra) foi feito por três anos a partir do terceiro ano de idade das plantas.

Para avaliação dos indicadores tecnológicos, procedeu-se o beneficiamento das castanhas dos 40 clones na fábrica-escola da Embrapa Agroindústria Tropical, que utiliza sistema semi mecanizado com autoclavagem à pressão de $2 \mathrm{kgf} . \mathrm{cm}^{-2}$, descorticação em máquinas de operação manual e estufagem a $55{ }^{\circ} \mathrm{C}$, seguida de despeliculagem manual (LIMA et al., 1995). As análises foram feitas em uma amostra com aproximadamente $3 \mathrm{~kg}$ de castanha dos clones da safra do ano de 2002.

Com base nos dados da performance de crescimento das plantas, produção de castanhas e indicadores tecnológicos da amêndoa foi feita a seleção dos clones pelo método em "tandem". Para Resende (2002), no sistema em "tandem", seleciona-se, por algumas gerações, para determinado caráter até atingir o nível desejado e, em gerações seguintes, para outros caracteres de interesse, dentre os descendentes dos indivíduos selecionados anteriormente. De acordo com Cruz \& Regazzi (1997), o índice de classificação ou índice com base em soma de posto "ranks" consiste em classificar os materiais genotípicos em relação a cada um dos caracteres, em ordem favorável ao melhoramento. Uma vez classificados, são somadas as ordens de cada material genético referente a cada caráter, resultando uma medida adicional tomada como índice de seleção.

A seleção com base no índice de classificação, metodologia de Mulamba \& Mock (1978), foi feita pela soma total dos pontos por cinco anos, considerando a soma de pontos do desempenho anual de cada clone para os caracteres de altura de planta, diâmetro de copa, peso médio e produção de castanha. Em seguida, foi feita a ordenação dos clones considerando somente o caráter produção de castanha pelo período de três anos.

Para altura de planta e diâmetro de copa a ordenação dos clones foi crescente, isto é, a seleção foi para clones com plantas mais baixas e menor envergadura de copa, enquanto que para o peso e produção de castanha a ordenação foi decrescente, ou seja, a seleção foi para clones com castanhas grandes e mais produtivos.

\section{RESULTADOS E DISCUSSÃO}

A análise de variância para os caracteres altura de planta (AP), diâmetro de copa (DC) e produtividade de castanha (PC) mostrou que não houve diferenças significativas entre clones, com exceção de DC em 2001, AP em 2002 e PC em 2004, provavelmente, devido à elevada intensidade de seleção para estas características que pode ter reduzido drasticamente o nível de variabilidade genética, indicando uma condição desfavorável à seleção futura para a redução do porte (Tabela 1). A média da altura de planta variou de $0,79 \mathrm{~m}$ no primeiro ano a 4,07 $\mathrm{m}$ no quinto ano, enquanto que para diâmetro de copa a variação foi de 0,71 $\mathrm{m}$ a $6,95 \mathrm{~m}$. A produtividade média dos 40 clones no quinto ano de idade das plantas, equivalente ao terceiro ano de avaliação da produção, foi de $976 \mathrm{~kg} \cdot \mathrm{ha}^{-1}$.

No melhoramento do cajueiro comum, tem-se buscado a seleção de plantas com porte e conformação de copa intermediária entre o tipo anão precoce e o comum, de modo que, pelo maior adensamento, sejam possíveis altas produtividades com menor tamanho de copa, embora haja necessidade de espaçamentos maiores nos plantio comerciais, em relação ao cajueiro anão precoce. $\mathrm{O}$ cajueiro comum possui porte elevado, com altura variando de $8 \mathrm{~m}$ a $15 \mathrm{~m}$ e envergadura de copa atingindo $20 \mathrm{~m}$. 
TABELA 1 - Quadrados médios de blocos (QMb), clones (QMc) e do resíduo (QMr) das análises de variância e respectivas significâncias; médias, valores máximos, mínimos (em metro) e coeficientes de variação experimental (CV) para altura de planta (AP), diâmetro de copa (DC) e produtividade de castanhas (PC - kg/ha) de 40 clones de cajueiro comum, do primeiro ao quinto ano de idade das plantas. Fazenda Jacaju (CIONE), Beberibe, CE.

\begin{tabular}{|c|c|c|c|c|c|c|c|c|c|c|c|}
\hline \multirow[t]{2}{*}{ F.V. } & \multicolumn{2}{|c|}{2000} & \multicolumn{2}{|c|}{2001} & \multicolumn{2}{|c|}{2002} & \multicolumn{2}{|c|}{2003} & \multicolumn{3}{|c|}{2004} \\
\hline & AP & DC & AP & DC & AP & DC & $\mathbf{A P}$ & DC & $\mathbf{A P}$ & DC & PC \\
\hline $\mathrm{QMb}$ & 0,0716 & 0,0092 & 0,1787 & 0,1606 & 0,4861 & 1,0584 & 0,5795 & 3,9981 & 0,4392 & 0,8294 & 187124,1 \\
\hline QMc & $0,0313^{\mathrm{NS}}$ & $0,0328^{\mathrm{NS}}$ & $0,0546^{\mathrm{NS}}$ & $0,2025^{*}$ & $0,2003 *$ & $0,3824^{\mathrm{NS}}$ & $0,3042^{\mathrm{NS}}$ & $0,7989^{\mathrm{NS}}$ & $0,2672^{\mathrm{NS}}$ & $1,0183^{\mathrm{NS}}$ & $115531,1 * *$ \\
\hline $\mathrm{QMr}$ & 0,0381 & 0,0455 & 0,0623 & 0,1733 & 0,1699 & 0,4179 & 0,2167 & 0,9468 & 0,2896 & 1,1959 & 58221,9 \\
\hline Média & 0,79 & 0,71 & 1,64 & 2,07 & 2,74 & 4,19 & 3,43 & 5,51 & 4,07 & 6,95 & 976,53 \\
\hline $\begin{array}{l}\text { Valor } \\
\text { máximo }\end{array}$ & 1,25 & 1,26 & 2,32 & 3,20 & 3,81 & 5,82 & 5,18 & 7,86 & 5,92 & 9,74 & $2.067,3$ \\
\hline $\begin{array}{l}\text { Valor } \\
\text { Mínimo }\end{array}$ & 0,35 & 0,23 & 1,04 & 1,08 & 1,78 & 2,75 & 2,28 & 3,32 & 2,80 & 4,58 & 439,75 \\
\hline $\mathrm{CV}(\%)$ & 24,6 & 29,9 & 15,2 & 20,1 & 15,0 & 15,44 & 13,6 & 17,65 & 13,2 & 15,7 & 24,7 \\
\hline
\end{tabular}

NS - não-significativo; * $\mathrm{p}<0,05 ; * *$ - $\mathrm{p}<0,01$.

Enquanto o tipo anão precoce caracteriza-se pelo porte baixo, altura abaixo de $4 \mathrm{~m}$, copa homogênea com variação no tamanho de 5,0 $\mathrm{m}$ a 6,5 m (BARROS, 1988).

No beneficiamento industrial da castanha houve tendência dos clones apresentarem bons indicadores industriais. A quase totalidade das castanhas (95\%) apresentou amêndoas classificadas como de alto peso $(>2,17 \mathrm{~g})$. Embora o rendimento industrial decresça com o aumento do peso das castanhas e, apesar dos clones possuírem apenas castanhas grandes $( \pm 14,80 \mathrm{~g})$ e médias $( \pm 9,70 \mathrm{~g})$, os mesmos apresentaram comportamento típico daqueles que possuem castanhas pequenas e médias, sendo $60 \%$ dos clones classificados como de alto rendimento (>23\%), 32,5\% como de médio rendimento (20-23\%) e apenas $7,5 \%$ dos clones se posicionaram na faixa de baixo rendimento industrial $(<20 \%)$ (Tabela 2$)$. A predominância de clones com alto rendimento industrial é explicada pelo elevado peso das amêndoas em relação ao peso das castanhas na maioria dos clones analisados.

Pela avaliação conjunta do porte das plantas, produção de castanha, performance fenotípica do clone e características tecnológicas da amêndoa, utilizando-se como referência a média da população dos 40 clones, elegeram-se os clones Comum 18, Comum 21, Comum 28, Comum 30, Comum 31 e Comum 36 como os mais promissores, até essa fase de desenvolvimento das plantas, com boas perspectivas para teste em larga escala e plantio comercial em cultivo de sequeiro na região Nordeste.

O peso médio da castanha variou de $9,9 \mathrm{~g}$ (Comum 30) a 17,1 g (Comum 21), enquanto que o peso médio da amêndoa foi menor para o clone Comum 30 (2,8 g) e maior para os clones Comum 31 e Comum 36, com 4,1 g. A variação no rendimento industrial para esses clones foi de 17 a $26 \%$ (Figura 1).

A maior produtividade de castanha nos dois primeiros anos de produção foi para o clone Comum 30, com 885 kg.ha-1 e $1.117 \mathrm{~kg} . \mathrm{ha}^{-1}$, respectivamente para o primeiro e segundo ano de produção, enquanto o clone Comum 18 obteve a menor produtividade dentre os seis mais produtivos (Figura 2). No terceiro ano a variação na produtividade foi de $980 \mathrm{~kg} \cdot \mathrm{ha}^{-1}$ a $1.299 \mathrm{~kg} \cdot \mathrm{ha}^{-1}$, respectivamente para os clones Comum 18 e Comum 21. $\mathrm{O}$ incremento na média de produtividade dos seis clones foi de $66 \%$ do $1^{\circ}$ para o $2^{\circ}$ ano e de $32 \%$ do $2^{\circ}$ para o $3^{\circ}$ ano. 
TABELA 2 - Distribuição de freqüência (\%) de 40 clones de cajueiro comum classificados pelos indicadores tecnológicos da castanha ${ }^{(1)}$.

\begin{tabular}{lccc}
\hline $\begin{array}{l}\text { Indicadores } \\
\text { tecnológicos }\end{array}$ & Alto & Médio & Baixo \\
\hline Peso das castanhas & 52,5 & 47,5 & - \\
$\begin{array}{l}\text { Peso das amêndoas } \\
\text { Rendimento industrial }\end{array}$ & 95,0 & 5,0 & - \\
$\begin{array}{l}\text { Índice de quebra das } \\
\text { amêndoas }\end{array}$ & 27,5 & 32,5 & 7,5 \\
$\begin{array}{l}\text { Relação bandas/ } \\
\text { amêndoas quebradas }\end{array}$ & 26,0 & 40,0 & 65,0 \\
$\begin{array}{l}\text { Índice de amêndoas } \\
\text { sadias }\end{array}$ & 20 & 20 & 60 \\
\hline
\end{tabular}

(1)Valores de referência, conforme Paiva et al. (2003).
Na Tabela 3 consta a somatória dos valores da classificação anual dos clones (r), tomando-se como referência os caracteres de altura de planta, diâmetro de copa, peso médio de castanha e produção de castanhas. A seleção com base na somatória dos r's no período de cinco anos, utilizando o mesmo critério do método em "tandem", ou seja, seleção de 15\% dos clones, alcançaria somente o clone Comum 31, com os demais clones distribuídos nas posições $10^{\circ}$, $17^{\circ}, 19^{\circ}, 21^{\circ}$ e $40^{\circ}$. Portanto, a seleção de clones de cajueiro comum utilizando essa metodologia diverge da seleção que considera o desempenho individual de cada clone, separadamente por característica. Quando a seleção foi feita considerando o valor dos r's anuais dos clones somente para o caráter produção de castanhas (Tabela 4), o quadro se apresentou diferente do anterior.

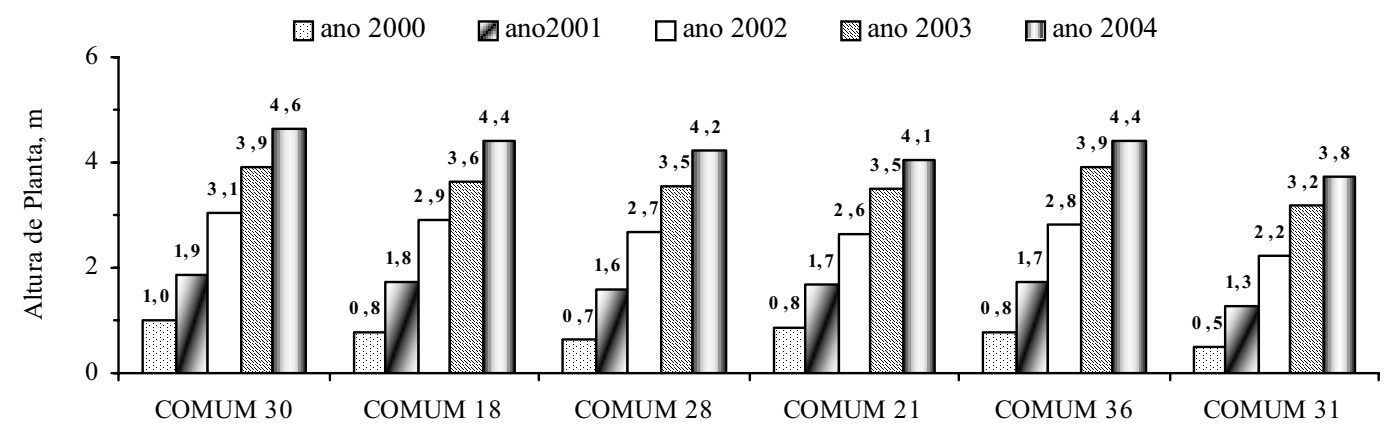

Clones

FIGURA 1 - Altura de planta de seis clones de cajueiro comum do primeiro ao quinto ano de idade das plantas, Fazenda Jacaju (CIONE), Beberibe, CE.

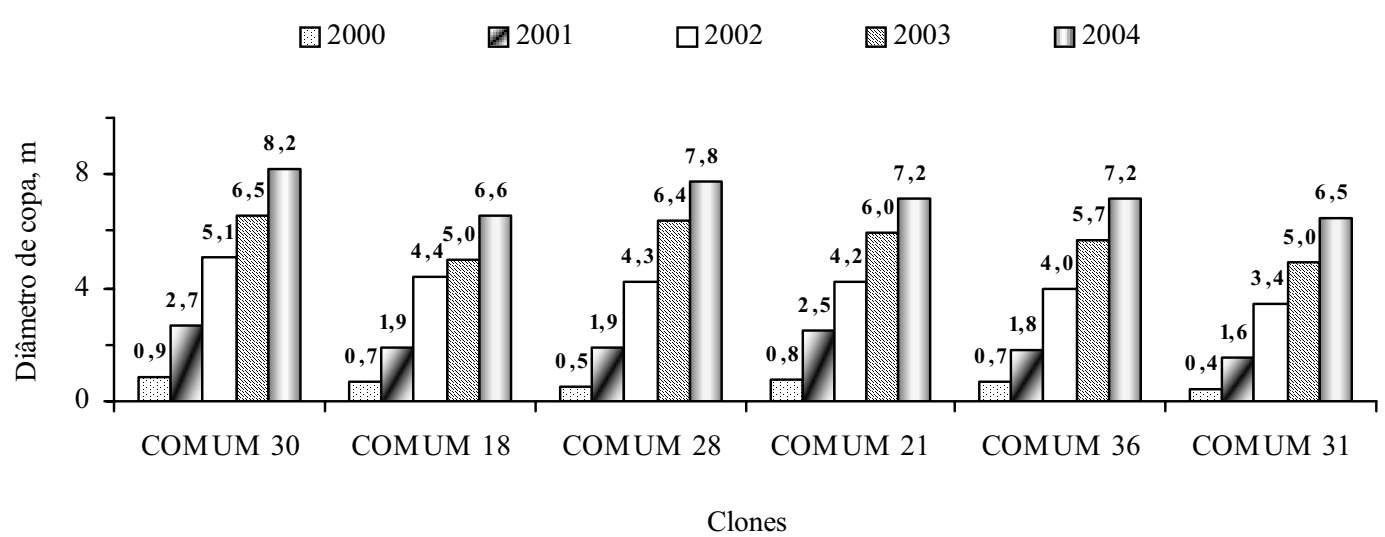

FIGURA 2 - Diâmetro de copa de seis clones de cajueiro comum do primeiro ao quinto ano de idade das plantas, Fazenda Jacaju (CIONE), Beberibe, CE. 
TABELA 3 - Somatória dos valores da classificação anual dos clones de cajueiro comum no período de cinco anos.

\begin{tabular}{|c|c|c|c|c|c|c|}
\hline \multirow{2}{*}{ Clone } & \multicolumn{5}{|c|}{ Soma anual dos r's } & \multirow{2}{*}{$\begin{array}{c}\text { Soma total } \\
\text { dos r's }\end{array}$} \\
\hline & 2000 & 2001 & 2002 & 2003 & 2004 & \\
\hline COMUM 31* & 2 & 2 & 22 & 20 & 20 & 66 \\
\hline COMUM 7 & 16 & 8 & 66 & 50 & 23 & 163 \\
\hline COMUM 12 & 35 & 7 & 47 & 37 & 38 & 164 \\
\hline COMUM 4 & 13 & 9 & 72 & 40 & 46 & 180 \\
\hline COMUM 22 & 9 & 15 & 60 & 52 & 45 & 181 \\
\hline COMUM 6 & 30 & 21 & 56 & 42 & 35 & 184 \\
\hline COMUM 13 & 30 & 12 & 60 & 39 & 53 & 194 \\
\hline COMUM 9 & 24 & 27 & 77 & 36 & 45 & 209 \\
\hline COMUM 8 & 55 & 39 & 67 & 30 & 24 & 215 \\
\hline COMUM 28 & 5 & 25 & 54 & 71 & 72 & 227 \\
\hline COMUM 11 & 50 & 35 & 72 & 38 & 46 & 241 \\
\hline COMUM 17 & 9 & 36 & 72 & 75 & 50 & 242 \\
\hline COMUM 3 & 66 & 34 & 56 & 40 & 50 & 246 \\
\hline COMUM 29 & 29 & 51 & 77 & 42 & 52 & 251 \\
\hline COMUM 5 & 24 & 42 & 78 & 64 & 61 & 269 \\
\hline COMUM 15 & 40 & 21 & 100 & 63 & 48 & 272 \\
\hline COMUM 36 & 32 & 38 & 63 & 65 & 77 & 275 \\
\hline COMUM 26 & 66 & 56 & 60 & 45 & 49 & 276 \\
\hline COMUM 18 & 28 & 43 & 92 & 52 & 62 & 277 \\
\hline COMUM 23 & 33 & 29 & 76 & 71 & 80 & 289 \\
\hline COMUM 21 & 60 & 62 & 45 & 63 & 62 & 292 \\
\hline COMUM 40 & 58 & 55 & 95 & 56 & 38 & 302 \\
\hline COMUM 34 & 48 & 68 & 73 & 52 & 66 & 307 \\
\hline COMUM 37 & 63 & 58 & 81 & 69 & 38 & 309 \\
\hline COMUM 39 & 56 & 36 & 84 & 67 & 80 & 323 \\
\hline COMUM 1 & 37 & 24 & 104 & 80 & 78 & 323 \\
\hline COMUM 16 & 62 & 54 & 92 & 40 & 75 & 323 \\
\hline COMUM 20 & 57 & 55 & 79 & 66 & 67 & 324 \\
\hline COMUM 24 & 14 & 40 & 91 & 83 & 104 & 332 \\
\hline COMUM 14 & 60 & 35 & 101 & 70 & 66 & 332 \\
\hline COMUM 38 & 43 & 38 & 99 & 78 & 84 & 342 \\
\hline COMUM 33 & 36 & 52 & 101 & 75 & 96 & 360 \\
\hline COMUM 10 & 40 & 52 & 93 & 109 & 67 & 361 \\
\hline COMUM 32 & 23 & 58 & 114 & 89 & 88 & 372 \\
\hline COMUM 19 & 33 & 60 & 139 & 82 & 75 & 389 \\
\hline COMUM 25 & 80 & 74 & 117 & 69 & 56 & 396 \\
\hline COMUM 2 & 77 & 73 & 110 & 74 & 72 & 406 \\
\hline COMUM 35 & 62 & 64 & 110 & 88 & 89 & 413 \\
\hline COMUM 27 & 59 & 52 & 110 & 100 & 93 & 414 \\
\hline COMUM 30 & 76 & 80 & 115 & 78 & 90 & 439 \\
\hline
\end{tabular}

* - clones selecionados pelo método em "tandem" em negrito. 
TABELA 4 - Classificação anual (r) dos clones de cajueiro comum para produção de castanha (PC), em ordem decrescente, pelo período de três anos.

\begin{tabular}{|c|c|c|c|c|c|c|c|c|}
\hline \multirow[b]{2}{*}{ Clone } & \multicolumn{2}{|r|}{2002} & \multirow[b]{2}{*}{ Clone } & \multicolumn{2}{|r|}{2003} & \multirow[b]{2}{*}{ Clone } & \multicolumn{2}{|r|}{2004} \\
\hline & $\mathbf{r}$ & $\begin{array}{c}\text { PC** } \\
\left(\text { kg.ha }^{-1}\right)\end{array}$ & & $\mathbf{r}$ & PC (kg.ha $\left.{ }^{-1}\right)$ & & $\mathbf{r}$ & PC $\left(\mathrm{kg} \cdot \mathrm{ha}^{-1}\right)$ \\
\hline COMUM 30* & 1 & 884,60 & COMUM 30 & 1 & 1117,3 & COMUM 10 & 1 & 1296,8 \\
\hline COMUM 21 & 2 & 800,05 & COMUM 36 & 2 & 947,4 & COMUM 40 & 2 & 1275,0 \\
\hline COMUM 5 & 3 & 701,90 & COMUM 5 & 3 & 938,7 & COMUM 17 & 3 & 1270,2 \\
\hline COMUM 16 & 4 & 594,50 & COMUM 21 & 4 & 922,1 & COMUM 28 & 4 & 1235,1 \\
\hline COMUM 31 & 5 & 524,75 & COMUM 31 & 5 & 873,3 & COMUM 31 & 5 & 1204,9 \\
\hline COMUM 26 & 6 & 459,90 & COMUM 16 & 6 & 839,5 & COMUM 8 & 6 & 1200,9 \\
\hline COMUM 9 & 7 & 443,00 & COMUM 29 & 7 & 781,5 & COMUM 5 & 7 & 1164,7 \\
\hline COMUM 23 & 8 & 436,40 & COMUM 28 & 8 & 776,9 & COMUM 29 & 8 & 1138,1 \\
\hline COMUM 13 & 9 & 388,25 & COMUM 35 & 9 & 727,4 & COMUM 15 & 9 & 1134,5 \\
\hline COMUM 20 & 10 & 361,90 & COMUM 23 & 10 & 710,5 & COMUM 7 & 10 & 1128,3 \\
\hline COMUM 17 & 11 & 359,50 & COMUM 9 & 11 & 697,2 & COMUM 35 & 11 & 1124,6 \\
\hline COMUM 3 & 12 & 356,00 & COMUM 18 & 12 & 694,5 & COMUM 30 & 12 & 1106,7 \\
\hline COMUM 28 & 13 & 353,75 & COMUM 33 & 13 & 670,6 & COMUM 36 & 13 & 1101,2 \\
\hline COMUM 36 & 14 & 341,60 & COMUM 26 & 14 & 660,1 & COMUM 21 & 14 & 1081,2 \\
\hline COMUM 8 & 15 & 322,00 & COMUM 20 & 15 & 657,3 & COMUM 37 & 15 & 1062,9 \\
\hline COMUM 14 & 16 & 320,00 & COMUM 13 & 16 & 647,0 & COMUM 18 & 16 & 1049,9 \\
\hline COMUM 18 & 17 & 308,60 & COMUM 17 & 17 & 639,7 & COMUM 23 & 17 & 1026,6 \\
\hline COMUM 2 & 18 & 299,50 & COMUM 14 & 18 & 628,1 & COMUM 1 & 18 & 1016,1 \\
\hline COMUM 1 & 19 & 298,33 & COMUM 1 & 19 & 617,7 & COMUM 14 & 19 & 1012,4 \\
\hline COMUM 10 & 20 & 297,50 & COMUM 8 & 20 & 609,0 & COMUM 9 & 20 & 1003,4 \\
\hline COMUM 25 & 21 & 288,60 & COMUM 3 & 21 & 591,5 & COMUM 39 & 21 & 998,4 \\
\hline COMUM 6 & 22 & 233,00 & COMUM 24 & 22 & 561,3 & COMUM 13 & 22 & 985,9 \\
\hline COMUM 34 & 23 & 228,75 & COMUM 32 & 23 & 553,8 & COMUM 25 & 23 & 966,9 \\
\hline COMUM 32 & 24 & 217,00 & COMUM 25 & 24 & 543,7 & COMUM 27 & 24 & 962,7 \\
\hline COMUM 7 & 25 & 209,17 & COMUM 37 & 25 & 542,7 & COMUM 20 & 25 & 936,7 \\
\hline COMUM 22 & 26 & 205,67 & COMUM 4 & 26 & 504,5 & COMUM 26 & 26 & 920,5 \\
\hline COMUM 11 & 27 & 204,75 & COMUM 11 & 27 & 500,5 & COMUM 4 & 27 & 918,8 \\
\hline COMUM 35 & 28 & 204,20 & COMUM 34 & 28 & 497,3 & COMUM 3 & 28 & 913,1 \\
\hline COMUM 40 & 29 & 194,30 & COMUM 15 & 29 & 487,3 & COMUM 2 & 29 & 906,1 \\
\hline COMUM 24 & 30 & 179,17 & COMUM 40 & 30 & 484,5 & COMUM 33 & 30 & 904,0 \\
\hline COMUM 12 & 31 & 163,00 & COMUM 12 & 31 & 482,3 & COMUM 12 & 31 & 848,3 \\
\hline COMUM 37 & 32 & 154,20 & COMUM 39 & 32 & 469,8 & COMUM 16 & 32 & 807,1 \\
\hline COMUM 4 & 33 & 136,67 & COMUM 2 & 33 & 460,4 & COMUM 6 & 33 & 785,7 \\
\hline COMUM 33 & 34 & 135,00 & COMUM 10 & 34 & 438,5 & COMUM 34 & 34 & 753,5 \\
\hline COMUM 29 & 35 & 100,83 & COMUM 7 & 35 & 429,7 & COMUM 19 & 35 & 688,7 \\
\hline COMUM 39 & 36 & 93,33 & COMUM 6 & 36 & 385,8 & COMUM 38 & 36 & 681,6 \\
\hline COMUM 38 & 37 & 88,25 & COMUM 38 & 37 & 382,4 & COMUM 32 & 37 & 651,6 \\
\hline COMUM 27 & 38 & 65,88 & COMUM 22 & 38 & 360,3 & COMUM 22 & 38 & 635,4 \\
\hline COMUM 15 & 39 & 0 & COMUM 19 & 39 & 346,0 & COMUM 11 & 39 & 615,6 \\
\hline COMUM 19 & 40 & 0 & COMUM 27 & 40 & 345,9 & COMUM 24 & 40 & 544,9 \\
\hline
\end{tabular}

Ciênc. agrotec., Lavras, v. 31, n. 3, p. 765-772, maio/jun., 2007 
Foram selecionados três, quatro e dois clones, respectivamente no $1^{\circ}, 2^{\circ}$ e $3^{\circ}$ ano de produção, com os demais clones selecionados pelo método em "tandem" distribuídos em posições intermediárias.

A aplicação da metodologia de Mulamba \& Mock (1978), descrita por Cruz \& Regazzi (1997), no melhoramento do cajueiro comum não obteve resultados satisfatórios, comparada à seleção pelo método em "tandem", devido ao mecanismo da seleção de planta envolver além de características quantitativas àquelas relacionadas à qualidade da amêndoa e a performance fenotípica da planta no campo. Normalmente, essas características são de difícil mensuração e dependem da experiência do selecionador.

Conforme Resende (2002), quando a seleção envolve mais de um caráter, três sistemas de seleção podem ser adotados: seleção em "tandem", níveis independentes de eliminação e índice de seleção. No primeiro, seleciona-se para determinado caráter até atingir o nível desejado e, em seguida, para outros caracteres de interesse. No segundo, níveis mínimos são estabelecidos para cada característica e todos os indivíduos abaixo deste nível são eliminados. No sistema índice de seleção considera-se simultaneamente todos os caracteres de interesse. Numa simulação que considera "n" caracteres de igual importância (mesmos valores econômicos, herdabilidades e variação fenotípica), é esperado que o índice de seleção seja superior aos outros sistemas, enquanto que o sistema de níveis independentes seja mais eficiente que o método em "tandem".

Apesar da facilidade e praticidade na utilização da metodologia de Mulamba \& Mock (1978), sua aplicação no melhoramento do cajueiro revelou-se limitada, contrariando ao que Paiva et al. (2002) e Silva et al. (2003) relataram no melhoramento da acerola e da batata, respectivamente.

\section{CONCLUSÕES}

Existe potencial de seleção de clones de cajueiro comum com produtividade acima de $1.500 \mathrm{~kg} \cdot \mathrm{ha}^{-1}$, castanhas com peso acima de $12 \mathrm{~g}$ e amêndoas com peso superior a 2,5 g, em cultivo de sequeiro.

Os clones Comum 18, Comum 21, Comum 28, Comum 30, Comum 31 e Comum 36 foram selecionados para teste em larga escala. É possível recomendá-los para o plantio comercial de sequeiro, em pequena escala (nível local), na região do município de Beberibe, CE.
A seleção de clones de cajueiro comum através do índice da soma de classificação revelou-se inapropriada comparada ao método em "tandem".

\section{AGRADECIMENTO}

À Cia. de Óleos Vegetais do Nordeste (CIONE), por permitir a instalação e viabilizar, até o momento, a manutenção do experimento de competição de clones de cajueiro comum na Fazenda Jacaju, em parceria com a Embrapa Agroindústria Tropical.

\section{REFERÊNCIAS BIBLIOGRÁFICAS}

BARROS, L. M. Melhoramento. In: LIMA, V. P. M. S. (Ed.). A cultura do cajueiro no Nordeste do Brasil. Fortaleza: BNB/ETENE, 1988. p. 321-356.

CRUZ, C. D.; REGAZZI, A. J. Modelos biométricos aplicados ao melhoramento genético. 2. ed. Viçosa: UFV, 1997. $390 \mathrm{p}$

FAO. FAOSTAT agriculture data. Disponível em: <http:// faostat.fao.org/faostat/collections?version= ext\&hasbulk=0\& subset=agriculture $>$. Acesso em: $25 \mathrm{fev}$. 2005.

LEITE, L. A. S.; PAULA-PESSOA, P. F. A. Aspectos sócioeconômicos. In: BARROS, L. M. (Ed.). Caju: produção: aspectos técnicos. Fortaleza: Embrapa Agroindústria Tropical; Brasília, DF: Informação Tecnológica, 2002. p. 15-17.

LIMA, A. C.; CHAGAS, F. A.; PAIVA, F. F. A.; PAULAPESSOA, P. F. A.; LEITE, L. A. S. Análise técnico-econômica de uma micro-unidade de processamento de castanha de caju. Fortaleza: [s.n.], 1995. 12 p.

MULAMBA, N. N.; MOCK, J. J. Improvement of yield potential of the method Eto Blanco maize (Zea mays L.) population by breeding for plant traits. Egyptian Journal of Genetics and Cytology, Alexandria, v. 7, p. 40-51, 1978.

OLIVEIRA, F. N. S. (Ed.). Sistema de produção para manejo do cajueiro comum e recuperação de pomares improdutivos. Fortaleza: Embrapa Agroindústria Tropical, 2004. 37 p. (Sistemas de Produção, 2). 
PAIVA, J. R.; ALVES, R. E.; MELO, F. I. O.; CORDEIRO, E. R.; ALMEIDA, A. S. Genetic progress of selections between and within caribbean cherry open pollination progenies. Crop Breeding and Applied Biotechnology, Londrina, v. 2, p. 299-306, 2002.

RESENDE, M. D. V. de. Genética biométrica e estatística no melhoramento de plantas perenes.
Brasília, DF: Embrapa Informação Tecnológica, 2002. $975 \mathrm{p}$.

SILVA, R. V.; SIMON, G. A.; PINTO, C. A. B. P. Seleção de híbridos interespecíficos de batata em programa de retrocruzamento. In: CONGRESSO BRASILEIRO DE MELHORAMENTO DE PLANTAS, 2., 2003, Porto Seguro, BA. Anais... Porto Seguro: [s.n.], 2003. 